Hoken Butsuri, 27, 135-142 (1992)

\title{
ORIGINAL
}

\section{Assessment of Initial Alveolar Deposition on Rats Exposed to Plutonium Aerosols Using a Whole Body Counter}

\author{
Nobuhito Ishigure, ${ }^{*, * 1}$ Takashi NaKano, ${ }^{* *, * 1}$ Hiroko EnOMOto, ${ }^{* * *, * 1}$ \\ Satoshi FuKuda, ${ }^{* * * *, * 1}$ Haruzo IIDA, ${ }^{* * * * *, * 1}$ Yoichi OGHISO,,$+* 1$ \\ Yutaka Yamada $\dagger^{+* * 1}$ and Jiro INABA $\dagger^{+} \dagger^{* * 1}$
}

(Received February 18, 1992)

(Accepted April 6, 1992)

In radiobiological studies on inhaled plutonium in experimental animals, radioactivity initially deposited on alveolar region is required for calculating absorbed dose and therefore determining dose-response relationship. To reduce the error related to variation within the sample of animals we intend to use in vivo detection of plutonium by whole body counting procedures to determine the initial alveolar deposition in each animal. The periodical in vivo counting also permits us to obtain an accurate retention curve with a small number of animals. It is concluded that the in vivo detection developed in the present work provides a powerful means to determine the initial alveolar deposition of the plutonium-exposed rats.

KEY WORDS: initial alveolar deposition, rats, plutonium, aerosols, whole body counter

\section{INTRODUCTION}

Plutonium is an important material in radiobiology and radiation protection. Inhalation of airborne particles of plutonium is the most important route of intake of this material. In a few developed countries, especially in USA, researches on inhalation toxicology of plutonium have been advanced on a large scale. ${ }^{1,2)}$ We, in National Institute of Radiological Sciences, have also started biological studies associated with plutonium involving metabolism, dosimetry, pathological effect and decorporation of the material so that we can obtain our own basic data required to assess human risks of internal exposure to plutonium.

* 石榑信人, $* *$ 仲野高志, $* * *$ 榎本宏子, $* * * *$ 福田 俊, ***** 飯田治三, †小木兽洋一, †十山田裕, †十稲葉次 郎

*1 放射線医学総合研究所内部被ばく研究部; 千葉市稲毛区 穴川 4-9-1 (而263)

Division of Comparative Radiotoxicology, National Institute of Radiological Sciences; 4-9-1, Anagawa, Inage-ku, Chiba 263, Japan.
Radioactivity initially deposited on alveolar region must be evaluated to calculate absorbed dose that determines dose-response relationship. For a group of animals exposed together in one batch of inhalation procedure the deposited radioactivity is usually estimated by liquid-scintillation counting of the autopsied lungs of the animals sacrificed as representative of the group. Though the liquid-scintillation counting enables precise assessment of the sacrificed animal itself, it is open to doubt whether the sacrificed animals truly represent the experimental group.

We intend to use in vivo detection of plutonium by whole body counting procedures to determine the initial alveolar deposition in each animal, which reduces the error related to variation within the sample of animals. The periodical in vivo counting also permits us to obtain an accurate retention curve with a small number of animals. In these procedures LX-rays emitted from the daughter products of plutonium isotopes are detected. The energies of the photons are very low and their number is small; therefore, a highly specialized system is required for useful results. 


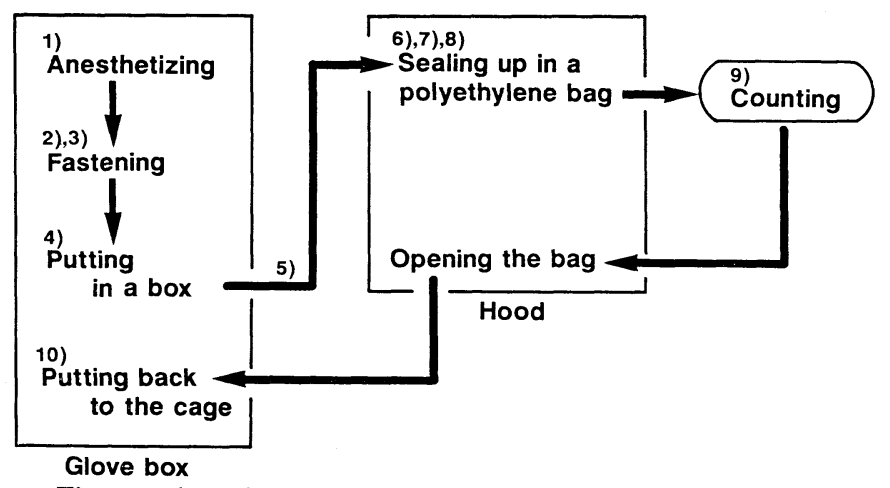

Fig. 1 Flow chart of the whole body counting procedures.

The present work describes the method for in vivo detection developed for small animals such as rats and a few results obtained from application of this technique to inhalation experiments.

\section{MATERIALS AND METHODS}

\section{Procedures of in vivo Detection}

According to a general rule of radiation protection, samples contaminated with significant activity of plutonium must be kept sealed up whenever they are handled in places other than a glove box or a hood. Handling a plutoniumburdened rat is not an exception to this regulation, even though the plutonium is contained within the rat body. However, we cannot use a thick and massive airtight box to contain the rat during counting, because the LX-rays, emitted at a small rate, are heavily attenuated owing to absorption by the thick walls of the box and to the detector-sample distance spaced by the wall. It makes the counting more difficult that the sample is a living animal that always requires air supply and moves if the body is not fixed.

Figure 1 shows a flow chart of the technique that was specially designed to solve these difficulties:

1) Anesthetizing an exposed rat in a glove box with a combination of ketamine hydrochloride and xylazine hydrochloride,

2) Laying down the rat flat on a thin plastic plate that is cut into the size of the rat,

3) Stretching the forelegs of the rat and fastening them with belts in order to fix the rat and expose its thorax to detector windows,

4) Putting the rat in a plastic box that is ad- iusted to the size of the rat: $230 \mathrm{~mm}(\mathrm{~W}) \times 51 \mathrm{~mm}$ (D) $\times 45 \mathrm{~mm}(\mathrm{H})$; the box is made of $2 \mathrm{~mm}$ thick "Lucite" plate and not airtight,

5) Taking out the box from the glove box through a double lid system and carrying it into a hood with an airtight bucket,

6) Putting the plastic box into a polyethylene bag with fastener,

7) Blowing air into the bag very gently with a small air pump so as to keep the rat alive during counting,

8) Zipping the bag to seal it up and wrapping the bag with another polyethylene bag,

9) Carrying the rat to a whole body counter,

10) After counting, usually for $1,000 \mathrm{~s}$, returning the rat to the cage in the glove box.

\section{A Whole Body Counter}

Figure 2 shows a schematic diagram of the whole body counter developed in the present work. Three thin NaI (T1) scintillation counters, $51 \mathrm{~mm}$ ( 2 inches) in diameter and $1 \mathrm{~mm}$ in thickness with an $\mathrm{Al}$ window of $6.9 \mathrm{mg} / \mathrm{cm}^{2}$, are housed within a lead box that has $50 \mathrm{~mm}$ thick low background lead shielding lined with plates of copper and acrylic resin. LX-rays that escape from the rat are counted by the detectors located at the ventral and the lateral sides of the rat. The pulse-amplitude distributions obtained with these three detectors are measured separately by feeding the output of each detector to a multichannel pulse height analyzer.

\section{RESULTS AND DISCUSSION}

\section{1. in vivo Detection}

The in vivo detection system is as follows. 


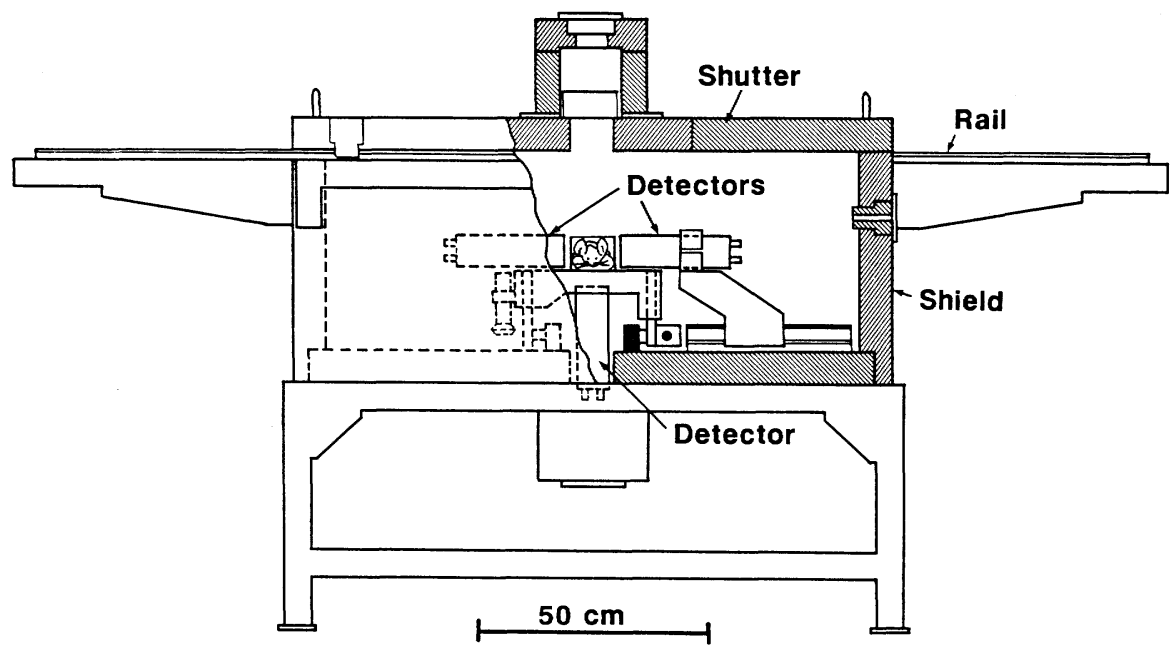

Fig. 2 Schematic diagram of the whole body counter.

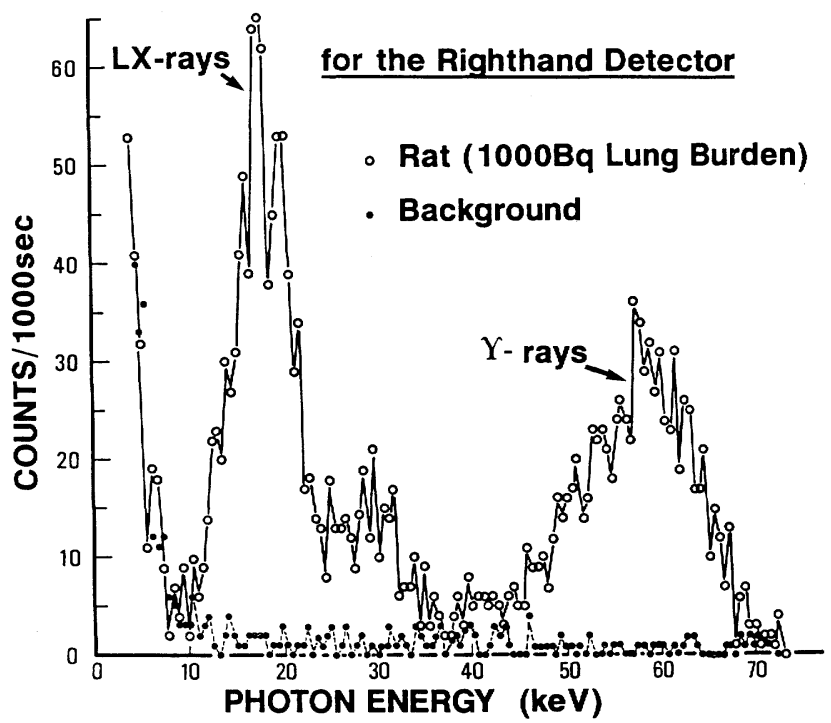

Fig. 3 An example of the energy spectrum of radiations from inhaled plutonium particles measured by the whole body counter. The lung burden of the sample was $1,000 \mathrm{~Bq}$.

\section{(1) Energy spectrum}

Figure 3 shows a typical energy spectrum obtained with the whole body counter. The lung burden of the sample was estimated to be 1,000 $\mathrm{Bq}$ by the present whole body counting. The spectrum exhibits two peaks at $17 \mathrm{keV}$ and 60 $\mathrm{keV}$. The peak at $17 \mathrm{keV}$ resulted from LX-rays emitted mainly from plutonium isotopes and the peak at $60 \mathrm{keV}$ from the $\gamma$-rays emitted from ${ }^{241} \mathrm{Am}$ as radioactive impurities in the plutonium source. In this figure the $\gamma$-rays gave comparable count rate to the LX-rays, though the $\gamma$-rays were emitted at a rate of one fourth for the LX-rays in the present source. This is because the LXrays were absorbed more heavily by the rat tissue than the $\gamma$-rays.

The total background counts of the three detectors, when an unexposed rat was loaded, were $0.26 \mathrm{cps}$ for LX-ray counting and $0.16 \mathrm{cps}$ for $\gamma$-ray counting. 

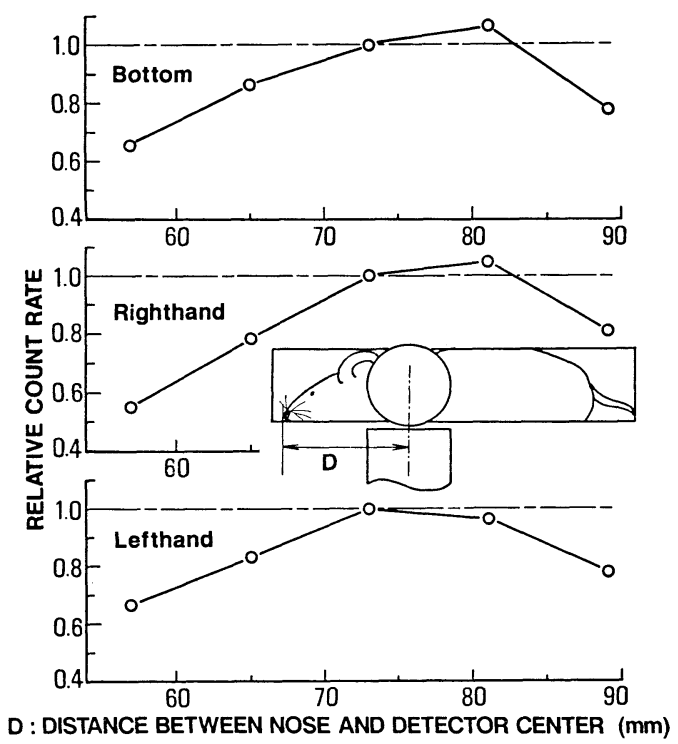

Fig. 4 Dependence of count rate on the rat-detector Eeometry with different locations of the rat along its longitudinal axis.

\section{(2) Geometry}

Since the LX-rays escaping from a rat are very weak, the detectors must be located close to the rat surface to enable a large counting efficiency. This, however, causes great variations of the counting efficiency owing to the changes in the geometry between the rat and the detectors.

An exposed rat was counted with different locations along its longitudinal axis. Figure 4 shows that the count rate was very susceptible to the locations of the rat; $5 \mathrm{~mm}$ difference brought $10 \%$ uncertainty to the determination of the lung burden. The count rate reached the maximum between 70 and $80 \mathrm{~mm}$ of the distance between the rat nose and the center of the detectors.

Figure 5 shows the result obtained when the height of the rat was changed. The abscissa shows the vertical distance between the ventral of the rat and the center of the detectors at the lateral side. The count rate reached maximum at the distances between 23 to $30 \mathrm{~mm}$. Since the rat can be placed precisely in the vertical direction, the uncertainty caused by the variation of the heights of the rat can be neglected.

(3) Interference by the plutonium at the parts other than in the lung

The body surface of an exposed rat might be contaminated with the plutonium that initially

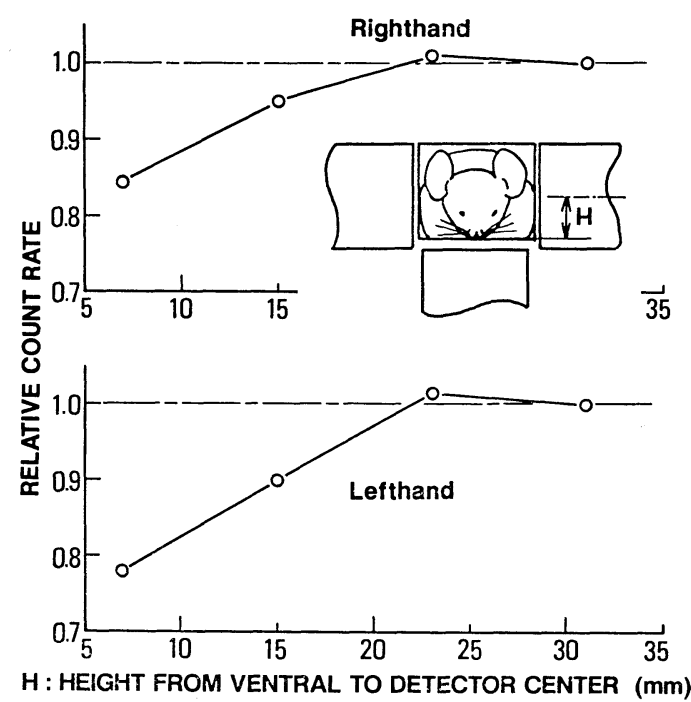

Fig. 5 Dependence of count rate on the rat-detector geometry with different locations of the rat in the up/down direction.

deposited around the nose, even though the surface around the nose was wiped with wet gauze immediately after exposure. The hair and skin might also be contaminated with plutonium-containing excreta.

The gastrointestinal (GI) tract retains much plutonium for a few days after exposure, because most plutonium deposited at the upper respiratory tract is mucociliarily transported very fast and swallowed into the stomach.

The LX-ray counts caused by these plutonium isotopes at the body surface and in the GI tract must be evaluated and subtracted from the total LX-ray counts so that we can assess the plutonium retained only in the lung.

Five exposed rats were sacrificed and their lungs taken out at two days after exposure. The remaining carcass was counted with the whole body counter to evaluate the plutonium retained at the parts other than in the lung. The count rate was compared with the whole body counting immediately before the sacrifice. The result is shown in Table 1, which clearly proved that the plutonium neither on the body surface nor in the GI tract affected the assessment of the initial alveolar deposition with our in vivo detection system, if at least 2 days pass after the exposure.

(4) Calibration

To calibrate our in vivo detection system, rats 
Table 1 Effect of the plutonium retained in the parts other than the alveolar region in whole body counting.

\begin{tabular}{cccc}
\hline Rat & Intact (I) (cps) & Without lung (W) (cps) & $W / I$ \\
\hline 1 & 5.68 & 0.06 & 0.011 \\
2 & 4.34 & 0.21 & 0.048 \\
3 & 3.47 & 0.00 & 0 \\
4 & 5.57 & 0.05 & 0.009 \\
5 & 3.46 & 0.04 & 0.012 \\
\hline
\end{tabular}

were sacrificed subsequently to whole body counting and the radioactivities of $\mathrm{Pu}$ isotopes and ${ }^{241} \mathrm{Am}$ in their autopsied lungs were measured with a phoswich type $\mathrm{NaI}(\mathrm{T} 1)$ scintillation detector. ${ }^{3)}$ The outline about the photon counting with the phoswich detector system is described in APPENDIX.

By using the radioactivity thus measured in the autopsied lung and the emission yields of LX-rays and $\gamma$-rays in each radioisotope, ${ }^{4)}$ the counting efficiencies both for LX-rays and $\gamma$-rays in the in vivo detection system were obtained. The result for LX-rays is shown in Fig. 6. Self-absorption differs with body weight, which varied the counting efficiency of LX-rays.

By using these counting efficiencies the radioactivity of plutonium in a living rat was obtained as follows:

1) The total emission rate of LX-rays was obtained by dividing the LX-ray counts by the LXray counting efficiency,

2) The activity of ${ }^{241} \mathrm{Am}$ was obtained by dividing the $\gamma$-ray counts by the $\gamma$-ray counting efficiency and by the $\gamma$-ray emission yield,

3) The emission rate of LX-rays from ${ }^{241} \mathrm{Am}$ alone was obtained by multiplying the activity of ${ }^{241} \mathrm{Am}$ by the LX-ray emission yield of ${ }^{241} \mathrm{Am}$.

4) The emission rate of LX-rays from $\mathrm{Pu}$ alone was obtained by subtracting the LX-ray emission rate due to ${ }^{241} \mathrm{Am}$ from the total LX-ray emission rate,

5) The radioactivity of plutonium was obtained by dividing the LX-ray emission rate from plutonium alone by the LX-ray emission yield of plutonium.

(5) Minimum detectable activity

The following formula gives the minimum detectable activity:

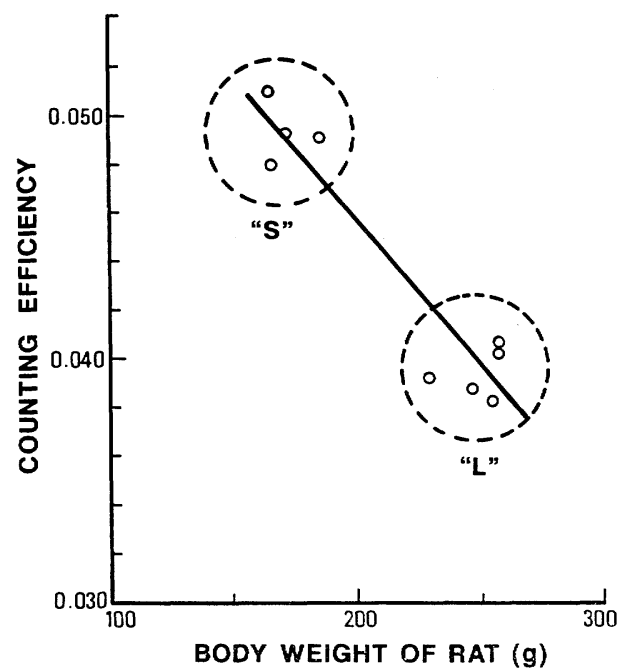

Fig. 6 Counting efficiency showing its dependence on body weight. They were obtained by using two different groups of body weights: the rats in the circle labeled " $\mathrm{S}$ " and those in the circle labeled "L." The average efficiency in each group is connected with a solid line.

$$
A=f \cdot\left\{\frac{1}{2 T E^{2}}+\frac{1}{2 E} \sqrt{\frac{1}{T^{2} E^{2}}+\frac{8}{T} B}\right\},
$$

where

$A$ : minimum detectable activity $(\mathrm{Bq})$,

$f$ : conversion coefficient $(=320 \mathrm{~Bq} / \mathrm{cps}$ for the rat of $170 \mathrm{~g}$ ),

$T$ : counting time $(=1,000 \mathrm{~s})$,

$B$ : count rate of background radiations (= $0.26 \mathrm{cps}$ )

$E$ : relative uncertainty due to statistics of count.

If $33 \%$ is allowed for the relative uncertainty, the minimum detectable activity was $23 \mathrm{~Bq}$, and for $10 \%$ relative uncertainty it was $91 \mathrm{~Bq}$.

\section{Initial Alveolar Deposition}

Figure 7 shows the measured distribution of initial alveolar deposition (IAD). The in vivo counting was performed at 7 to 15 days after exposure. The activity at the exposure day was obtained by extrapolating a measured retention curve to zero day.

The rats were trained for two weeks prior to exposure so that they autonomously stick their nose from the hole of the rat holder and tamely breathe plutonium-containing air without anesthetizing. The body weight of the rats in the groups " $A$ " and "B" was around $240 \mathrm{~g}$ and in 
Table 2 Deposition fractions of inhaled plutonium aerosols in alveolar region of rats.

\begin{tabular}{ccccccc}
\hline Group & AMAD*1 $_{(\mu \mathrm{m})}$ & GSD & Exposure*2 $(\mathrm{min} \cdot \mathrm{Bq} / \mathrm{ml})$ & $\mathrm{IAD}(\mathrm{Bq})$ & Resp. Vol.*3(ml $/ \mathrm{min})$ & Depos. Frac. $(\%)$ \\
\hline A & 0.47 & 2.1 & 69 & 2350 & 124 & 27 \\
B & 0.48 & 2.0 & 60 & 2310 & 128 & 30 \\
C & 0.44 & 2.0 & $38 \sim 45$ & $800^{* 4}$ & $(9$ & $18 \sim 21$ \\
D & 0.40 & 1.9 & 46 & 1060 & 99 & 23 \\
E & 0.43 & 2.0 & 86 & 1390 & 97 & 17 \\
\hline
\end{tabular}

*1 Activity median aerodynamic diameter.

*2 Time integral of air concentration of plutonium during exposure.

*3 Calculated values using the Guytonformula. ${ }^{7)}$

*4 Rejecting the three lowest IAD.
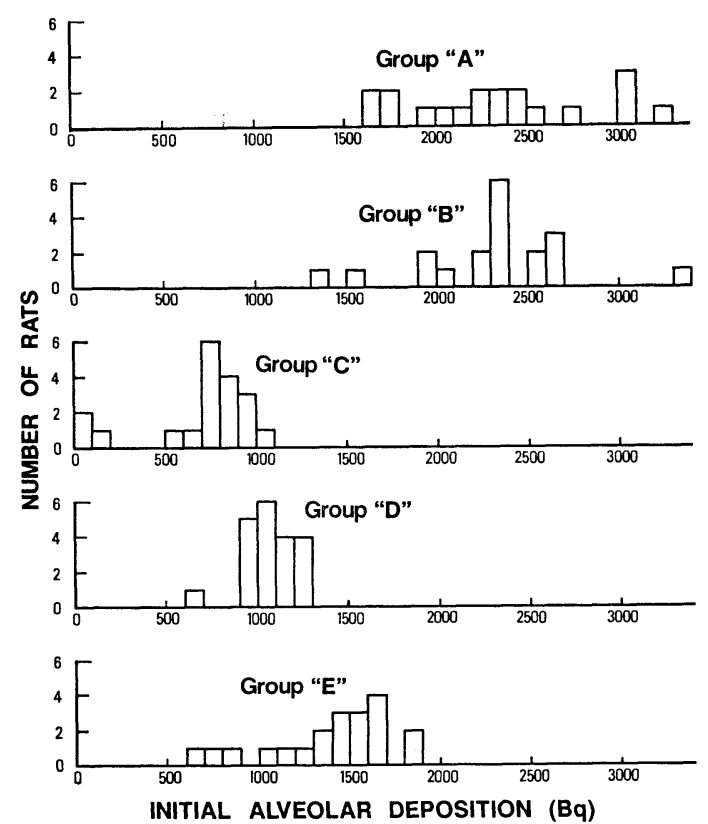

Fig. 7 Distributions of initial alveolar depositions of plutonium dioxide particles for the five different batches of exposure.

the groups "C" to "E" around $170 \mathrm{~g}$.

The present results proved that the IAD aimed in these exposures between 1,000 and $2,000 \mathrm{~Bq}$, which efficiently induces lung cancer in rats, ${ }^{1)}$ was mostly attained.

The relative standard deviation was between 13 and $24 \%$, if a few extraordinarily small IAD were rejected. These variations are not large compared with that reported by Pacific Northwest Laboratory, ${ }^{5)}$ a leading organization of the world that has carried out inhalation experiments on a large scale.
As found typically in the group "C," a few rats inhaled plutonium very little. The concentration of aerosols at the position of rat nose is almost uniform. ${ }^{6}$ These rats might be packed too tightly in a holder, which prevented the normal respiration, or they might be so loosely contained in the holder that they could easily move and pull in their nose from the hole of the holder for a long time.

The intra- and inter-group variations in IAD justify our plan that the IAD of individual rat should be measured one by one with in vivo counting so that we can determine accurate dose and a certain dose-response curve.

Though we did not measure the actual respiration of the rats during exposure, we tried to estimate the deposition fraction using the following equation:

Deposition fraction $(\%)=100 \times I A D /($ Exposure

$\times$ Respiratory volume per minute), where the "exposure" is a time integral of air concentration of plutonium $(\mathrm{min} \cdot \mathrm{Bq} / \mathrm{ml})$, which was determined by air sampling during exposure. The radioactivity collected on the air filters was measured by LX-ray counting with a phoswich detector. ${ }^{3)}$ Assuming that the respiratory volume of rats can be calculated using the Guyton formula ${ }^{7}$

Respiratory volume per minute $(\mathrm{ml})$ $=2.1 \times(\text { Body weight, } \mathrm{g})^{0.75}$,

the results as shown in Table 2 were obtained. Size distributions of plutonium aerosols are listed in the same tabel, which were determined with a cascade impactor. The radioactivity at each stage was also measured by LX-ray counting.

In the groups from " $C$ " to " $E$ " the deposition fractions between 17 and $23 \%$ were obtained. 
In the groups " $\mathrm{A}$ " and " $\mathrm{B}$," on the other hand, larger deposition fractions, between 27 and $30 \%$, were obtained. Since the size of the rats in the groups " $A$ " and " $B$ " were much different from that in the groups " $C$ " to " $E$," as stated above, the adaptability of the rats to the rat holder was not the same to each other, which might vary the respiration conditions. This inter-group difference might also be contributed by the uncertainty in the estimation of the respiratory volume using the Guyton formula.

There are very limited experimental data about particle deposition in laboratory animals. RAABE et $a l^{8)}$ measured regional deposition of monodisperse aerosols of fused aluminosilicate particles in rats. They used unanesthetized rats, too. Their exposure chamber was similar to that used in the present work. According to their data, the IAD for the particles of $0.44 \mu \mathrm{m}$ in AMAD was $13 \%$, which was smaller than our IAD values in any groups.

Among the mechanisms of particle deposition, sedimentation and impaction depend on the AM$\mathrm{AD}$ of the particle, whereas diffusion varies inversely proportional to the square root of the geometrical diameter. ${ }^{9}$ ) The geometrical diameter of the particles of $0.44 \mu \mathrm{m}$ in AMAD was 0.085 $\mu \mathrm{m}$ for plutonium dioxide and $0.26 \mu \mathrm{m}$ for fused aluminosilicate particles, which were obtained from the density of each material and the Cunningham slip correction factor. ${ }^{10}$ The deposition by diffusion is hence larger for plutonium-dioxide particle by 1.75 times $(=\sqrt{0.26 / 0.085})$ than for fused aluminosilicate. This might be one of the reasons why the IAD obtained in the present work was larger than that by RAABE et al.

As referred to the calculational model by $\mathrm{T}_{\mathrm{AKA}}$ HASHI, ${ }^{9}$ the deposition fractions are in the range between $24 \%$ (for the particle diameter of 0.085 $\mu \mathrm{m}$ ) and $13 \%$ (for $0.44 \mu \mathrm{m}$ ). For the groups from " $C$ " to " $E$," the present results were within this range, but for the groups " $A$ " and " $B$ " our values were beyond this range.

To obtain the deposition fraction with a higher accuracy, which is required for more detailed discussion, we have to collect the data on the actual respiration conditions of rats during exposure. This is the subject for a future study.

\section{CONCLUSION}

It is concluded that the in vivo detection with the whole body counter provides a powerful means to determine the initial alveolar deposition of the plutonium-exposed rats, which can reduce the error related to variation within the sample of animals and reduce sample size required in the animal experiment involving metabolism, dosimetry, biological effect and decorporation of the plutonium.

ACKNOWLEDGEMENTS: The authors wish to thank Dr. H. Sato of NIRS, Chiba for the preparing of the source solution for the plutonium aerosols, Mr. A. Koizumi, Dr. Y. Yamada, Mr. K. Miyamoto and Dr. S. Takahashi (all of NIRS, Chiba) for their help in the inhalation experiment.

\section{APPENDIX}

Calibration of phoswich detector system:

Since photon counting by a phoswich detector gives a relative measurement of radioactivity, the detection system has to be calibrated to obtain absolute radioactivity. The procedures are as follows:

1) for ${ }^{241} \mathrm{Am}$ the counting system was calibrated for $\gamma$-ray counts by using a commercially available standard source of ${ }^{241} \mathrm{Am}$,

2) the ratio of (LX-ray counts)/( $\gamma$-ray counts) of ${ }^{241} \mathrm{Am}$ (this ratio was denoted as $R$ ) was obtained by using this standard source,

3) an evaporated source was prepared from a source solution for plutonium aerosols,

4) the total $\alpha$-activity of this evaporated source was determined by a solid state nuclear track detector,

5) the activity of ${ }^{241} \mathrm{Am}$ in this source was measured by using a calibration curve formed in the first procedure,

6) this activity was subtracted from the total $\alpha$-activity of the evaporated source and then the $\alpha$-activity of plutonium isotopes in this source was obtained,

7) photon counting of this evaporated source was performed and to obtain the LX-ray counts from plutonium alone the LX-ray counts due to ${ }^{241} \mathrm{Am}(=R \times \gamma$-ray counts) was subtracted from the total LX-ray counts, 
8) a calibration curve was determined by the relation between this LX-ray counts and the $\alpha$ activity of plutonium,

9) an autopsied sample was counted with the phoswich detector and the LX-ray counts due to plutonium alone was obtained by the same manner as the evaporated source,

10) the $\alpha$-activity was determined by using the calibration curve formed in the procedure 8).

Detailed discussion about these procedures, for example, the problems of self-absorption, back scattering or geometrical changes will be reported in the other paper. ${ }^{3)}$

\section{REFERENCES}

1) C.L. Sanders, K.E. McDonald and J.A. MaHaFFEY; Lung tumor response to inhaled $\mathrm{Pu}$ and its implications for radiation protection, Health Phys., 55, 455 (1988).

2) R.O. MCClellan, B.B. Boecker, F.F. Hahn and B.A. Muggenburg; Lovelace ITRI studies on the toxicity of inhaled radionuclides in beagle dogs, in "Life-span Radiation Effects Studies in Animals: What Can They Tell Us? (R.C. THOMPson and J.E. MahaffeY, eds.)," p. 74 (1986), Office of Scientific and Technical Information.

3) N. Ishigure, T. Nakano and H. Enomoto; Non-destructive analysis of plutonium in bulk samples using a phoswich detector (in prepara- tion).

4) V.S.SHIRLEY ed.; "Tatle of Radioactive Isotores," John Wiley \& Sons, New York (1986).

5) W.C. Cannon; Aerosol technology development, in "Pacific Northwest Laboratory Annual Report for 1982 to the DOE Office of Energy Research, Part 1, Biomedical Sciences," p. 55 (1982), NTIS.

6) Y. Yamada, Y. Kubota, A. Koizumi and O. MATSUOKA; Design of radioactive aerosol exposure system for small rodents and its basic characteristics (in Japanese), Hoken Butsuri, 24, 331 (1989).

7) A.C. Guyton; Measurement of the respiratory volume of laboratory animals, Am. J. Physiol. 150, 70 (1947).

8) O.G. RaAbe, M.A. Al-Bayati, S.V. Teague and A. RASOLT; Regional deposition of inhaled monodisperse coarse and fine aerosol particles in small laboratory animals, in "Inhaled Particles" VI, ed. by J. Dodgson, R.I. McCallum, M.R. BAILeY and D.R. FISHER, p. 53 (1988), Pergamon Press, Oxford.

9) K. TaKahashi; Inhaled particle deposition in mammalian lungs-Study by calculational model -(in Japanese), J. Jpn. Soc. Air Pollut., 23, 349 (1988).

10) C.N. Davies; Definitive equations for the fluid resistance of spheres, Proc. Phys. Soc., 57, 259 (1945). 